\title{
Video Article \\ RNAi-mediated Control of Aflatoxins in Peanut: Method to Analyze Mycotoxin Production and Transgene Expression in the Peanut/Aspergillus Pathosystem
}

\author{
Renée S. Arias ${ }^{1}$, Phat M. Dang ${ }^{1}$, Victor S. Sobolev ${ }^{1}$ \\ ${ }^{1}$ National Peanut Research Laboratory, United States Department of Agriculture, Agricultural Research Service
}

Correspondence to: Renée S. Arias at renee.arias@ars.usda.gov

URL: https://www.jove.com/video/53398

DOI: doi:10.3791/53398

Keywords: Environmental Sciences, Issue 106, RNA interference, silencing, groundnut, seed, transgenic, Aspergillus

Date Published: $12 / 21 / 2015$

Citation: Arias, R.S., Dang, P.M., Sobolev, V.S. RNAi-mediated Control of Aflatoxins in Peanut: Method to Analyze Mycotoxin Production and Transgene Expression in the Peanut/Aspergillus Pathosystem. J. Vis. Exp. (106), e53398, doi:10.3791/53398 (2015).

\section{Abstract}

The Food and Agriculture Organization of the United Nations estimates that $25 \%$ of the food crops in the world are contaminated with aflatoxins. That represents 100 million tons of food being destroyed or diverted to non-human consumption each year. Aflatoxins are powerful carcinogens normally accumulated by the fungi Aspergillus flavus and $A$. parasiticus in cereals, nuts, root crops and other agricultural products. Silencing of five aflatoxin-synthesis genes by RNA interference (RNAi) in peanut plants was used to control aflatoxin accumulation following inoculation with A. flavus. Previously, no method existed to analyze the effectiveness of RNAi in individual peanut transgenic events, as these usually produce few seeds, and traditional methods of large field experiments under aflatoxin-conducive conditions were not an option. In the field, the probability of finding naturally contaminated seeds is often $1 / 100$ to $1 / 1,000$. In addition, aflatoxin contamination is not uniformly distributed. Our method uses few seeds per transgenic event, with small pieces processed for real-time PCR (RT-PCR) or small RNA sequencing, and for analysis of aflatoxin accumulation by ultra-performance liquid chromatography (UPLC). RNAi-expressing peanut lines 288-72 and 288-74, showed up to $100 \%$ reduction $(p \leq 0.01)$ in aflatoxin $B_{1}$ and $B_{2}$ compared to the control that accumulated up to $14,000 \mathrm{ng}^{-1}$ of aflatoxin $B_{1}$ when inoculated with aflatoxigenic $A$. flavus. As reference, the maximum total of aflatoxins allowable for human consumption in the United States is $20 \mathrm{ng}^{-1}$. This protocol describes the application of RNAi-mediated control of aflatoxins in transgenic peanut seeds and methods for its evaluation. We believe that its application in breeding of peanut and other crops will bring rapid advancement in this important area of science, medicine and human nutrition, and will significantly contribute to the international effort to control aflatoxins, and potentially other mycotoxins in major food crops.

\section{Video Link}

The video component of this article can be found at https://www.jove.com/video/53398/

\section{Introduction}

Approximately 4.5 billion people are chronically exposed to aflatoxins ${ }^{1}$, the most powerful carcinogens known in nature ${ }^{2}$. These mycotoxins contaminate $25 \%$ of the food crops in the world ${ }^{3}$, including maize, cassava, rice, nuts, cereals and spices. ${ }^{4}$. Aflatoxins cause stunting in children 5 , impair the immune system ${ }^{6}$, are present in $58 \%$ of hepatocellular-carcinomas in human biopsies ${ }^{7,8}$, and kill hundreds of people during periodic outbreaks of aflatoxicosis ${ }^{9,10}$. Aflatoxins are polyketide-derived mycotoxins normally produced by Aspergillus flavus and $A$. parasiticus; aflatoxins $B_{1}$ and $B_{2}$ are produced by $A$. flavus, whereas $A$. parasiticus also produces $G_{1}$ and $G_{2}$. The chemical structure of these compounds and a chromatogram showing their separation by UPLC are shown in Figure 1. 


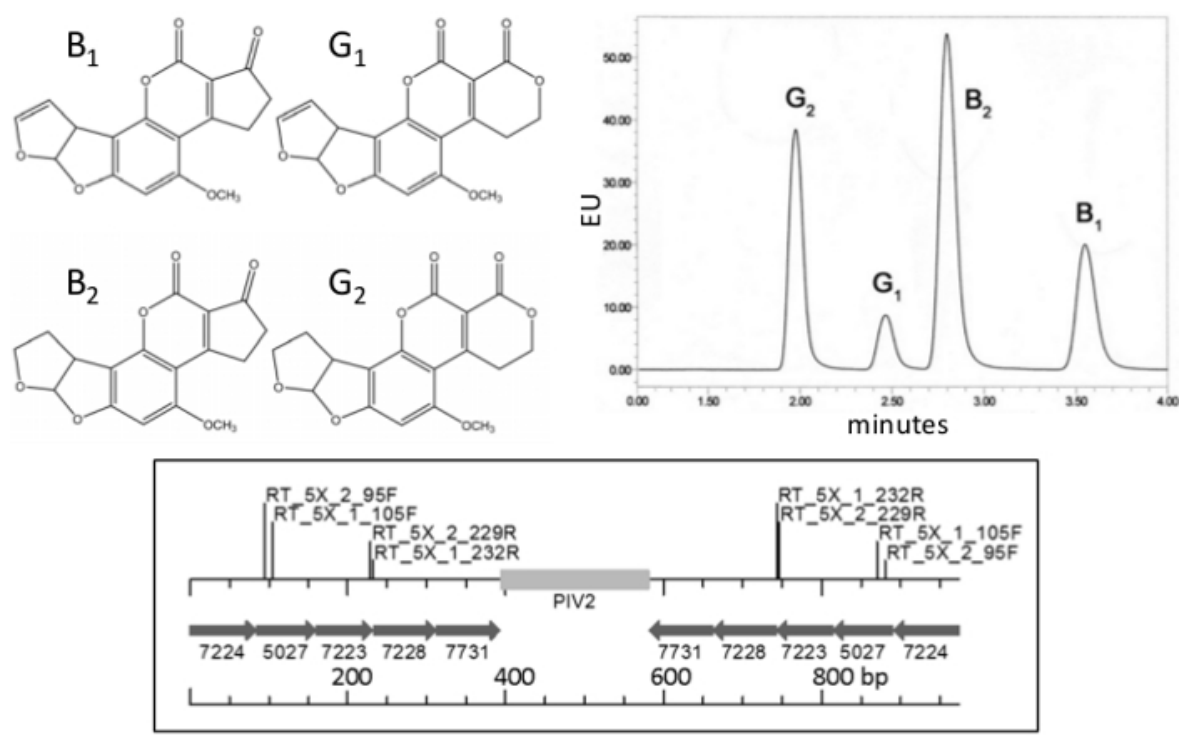

Figure 1. Aflatoxins and RNAi insert. Top: chemical structure (left) and example of chromatogram (right) of the four most common polyketidederived aflatoxins: $B_{1}, B_{2}, G_{1}$ and $G_{2}$, produced by Aspergillus parasiticus, $A$. flavus produces $B_{1}$ and $B_{2}$. Bottom: Schematic of gene fragments in the RNAi construct p5XCAPD used for peanut transformation, numbers under arrows are gene-fragment accession numbers in the Aspergillus flavus genome; PIV2: potato intron; bp: base pairs; RT_5X_1 and RT_5X_2: Real-Time PCR primer sites. Please click here to view a larger version of this figure.

Economic losses in exports due to aflatoxins in peanut alone exceed $\$ 450$ million U.S. dollars if calculated based on the $4 \mathrm{ng} \mathrm{g}^{-1}$ limit of aflatoxin allowed for human consumption in the European Union ${ }^{11}$. Aflatoxins have been known for 60 years ${ }^{12}$; however, though many agricultural practices were developed to mitigate their effect, including application of other fungal strains ${ }^{13,14}$, no consistent method of control exists, and resistant plant varieties are not available. Testing plant germplasm for resistance to aflatoxins is particularly difficult, because even under conducive conditions for pathogen invasion, mycotoxin accumulation is unpredictable and does not follow a normal distribution. Thus, experiments usually require large planting areas, hundreds of seeds and multiple samples of $100-1,700 \mathrm{~g}$ to reduce variability of the data ${ }^{15,16}$

RNA interference was discovered in $1998{ }^{17}$; and the benefits of "silencing" are currently being explored in a number of new applications, e.g. in human therapies against metastatic breast cancer ${ }^{18}$, liver cancer ${ }^{19}$, myeloid leukemia ${ }^{20}$, and in plant protection against insects ${ }^{21}$ and nematodes ${ }^{22}$. In plants, RNA interference signals can travel cell to cell, with small interfering RNA (siRNA) and high molecular weight RNA being responsible for the systemic posttranscriptional gene silencing ${ }^{23,24}$, even inside fungal pathogens that are in close contact with plant host ${ }^{25}$. The effectiveness of RNAi on plant-mediated silencing of fungal-pathogen genes has been described in few plant pathosystems, for these, visual examination of symptoms in the aerial parts of the plants (leaves) allowed disease quantification, i.e., oomycete Bremia in lettuce ${ }^{26}$, Puccinia in wheat ${ }^{27}$ and Fusarium in banana ${ }^{28}$. Much more difficult is to evaluate RNAi effectiveness to control mycotoxins in plants, particularly aflatoxins in peanuts as the leaves show no symptoms of infection, the organs invaded (seeds) are under several inches of soil, the occurrence of infection is unpredictable, and only chemical analysis can determine the presence of aflatoxins. In addition, each transgenic event in peanut normally produces few seeds (4-6 per plant); therefore, traditional testing for a no-aflatoxin accumulation trait in large field plots, lasting entire cropping seasons, and using hundreds of seeds is not feasible. A method is described here to analyze in less than one week, RNAi peanut seeds for presence of transgene and for a no-aflatoxin accumulation trait, using only few seeds.

\section{Protocol}

\section{Molecular Construct and Peanut Transformation}

1. Combine DNA fragments of five A. flavus genes, AFL2G_07223 (aflS or aflJ), AFL2G_07224 (aflR), AFL2G_07228 (aflC/pksA/pksL1), AFL2G_07731 (pes1) and AFL2G_05027 (aflatoxin efflux pump, aflep). For this, use the following primers and ultramers: DIR-1, Short-Dir1R, DIR-2-inverted, Short-Dir2-R, DirAll-Nco-Rv, and DirAll-BamEco-Fw, Table 1.

1. Make DIR-1 double strand by 5 PCR cycles $\left(25 \mu \mathrm{l}\right.$ reaction; $95^{\circ} \mathrm{C} 2 \mathrm{~min}$, followed by 5 cycles of $94{ }^{\circ} \mathrm{C} 45 \mathrm{sec}, 55^{\circ} \mathrm{C} 30 \mathrm{sec}, 68^{\circ} \mathrm{C} 15$ $\mathrm{sec}$ ) using DNA polymerase according to manufacturer's instructions and primer Short-Dir1-R to leave a $3^{\prime}$ overhang CCCGT. Repeat these steps to make DIR-2-inverted double strand using primer Short-Dir2-R to leave a 3' overhang ACGGG complementary to DIR-1.

2. Ligate the two 199 bp fragments with T4 DNA Ligase as per manufacturer's instructions. PCR amplify the resulting 393 bp fragment as indicated in 1.1.1 using primers DirAll-cacc-Fw and DirAll-Nco-Rv (Table 1), and clone the product using standard techniques into pENTR1A to make plasmid p2+4ENTR.

3. Recombine p2+4ENTR into pCAPD ${ }^{29}$ (NCBI Accession: KC176455.1) using LR clonase Il enzyme mix according to manufacturer's instructions to make plasmid p5XCAPD, and transform it into Escherichia coli DH5a using standard techniques followed by partial sequencing. Note: The complete RNAi insert is shown in Table 1.

2. Transform Agrobacterium strain $\mathrm{C} 58 \mathrm{C} 1{ }^{30}$ with plasmid $\mathrm{p} 5 \mathrm{XCAPD}$ as previously reported ${ }^{30}$, and use the resulting bacterium to transform peanut plants as follows: 
1. Grow at $30^{\circ} \mathrm{C}$ the Agrobacterium harboring p5XCAPD, use for this, $50 \mathrm{ml} \mathrm{LB-Broth} \mathrm{supplemented} \mathrm{with} 500 \mu \mathrm{g} \mathrm{ml}{ }^{-1}$ streptomycin, $25 \mu \mathrm{g}$ $\mathrm{ml}^{-1}$ gentamicin, $10 \mathrm{\mu g} \mathrm{ml}^{-1}$ kanamycin, and shake the culture at $250 \mathrm{rpm}$ until reaching $1 \mathrm{OD}_{260}$.

2. Harvest the Agrobacterium cells by centrifugation $(6,000 \times \mathrm{g})$ for $10 \mathrm{~min}$, resuspend in $50 \mathrm{ml} \mathrm{AB}$ minimal medium ${ }^{31}$ with $100 \mu \mathrm{M}$ acetosyringone for $1 \mathrm{hr}$, and place in the bacterial suspension the explants from 10-14 day old seedlings Exp27-1516, runner-type peanut breeding line. Blot dry the explants on 3MM blotting paper after $30 \mathrm{~min}$, and place them on shoot-induction medium (SIM) [MS salt ${ }^{32}, 3 \%$ sucrose, $20 \mu \mathrm{M}$ benzylaminopurine (BAP), $10 \mu \mathrm{M}$ thidiazuron (TDZ), $\mathrm{pH} 5.8,0.3 \%$ gellan gum] without antibiotics in the dark for three days.

3. Do tissue selection and regeneration as reported before ${ }^{33}$. Move tissues to SIM (500 $\mu \mathrm{M}$ cefotaxime and $100 \mu \mathrm{M}$ kanamycin) for shoot formation, with bi-weekly transfers for 2 months. Then place expanding shoots on shoot-elongation medium (SEM) [5 $\mu \mathrm{M}$ BAP, $1 \mu \mathrm{M}$ gibberellic acid $\left(\mathrm{GA}_{3}\right)$ ], bi-weekly for several months.

4. Place individual shoots, $2 \mathrm{~cm}$ in size, in root-induction medium (RIM) [1/2 MS, 1.5\% sucrose, $5 \mu \mathrm{M}$ a-naphthalene-acetic acid (NAA), $2.5 \mu \mathrm{M}$ indole-butyric acid (IBA)], then acclimate the seedlings and transfer them to the greenhouse.

\section{Identification of Peanut Plants Harboring RNAi to Silence Aflatoxin Synthesis Genes}

1. Use plant mini kit in a robot workstation with $200 \mu$ l elution according to manufacturer's instructions to extract DNA from young leaves of peanut plants that were subject to the process of transformation (as previously described) with RNAi construct p5XCAPD (Figure 1) which has as backbone plasmid pCAPD ${ }^{29}$ for gene silencing.

2. Screen the DNA samples by single-tube nested PCR (STN-PCR) as described previously ${ }^{34}$ to detect the selectable marker NPTII and the RNAi insert from p5XCAPD. Clonally propagate from cuts (3-4 nodes) PCR positive plants to produce sufficient seeds for testing in this first generation.

1. Use four 2-fold dilutions of DNA (50-100 $\mathrm{ng} \mu \mathrm{I}^{-1}$ before dilution) in all STN-PCR reactions. For NPTII, use external primers PCAPD 5714F: 5'-AGGCTATTCGGCTATGACTG-3' and PCAPD 6446R: 5'-CGTCAAGAAGGCGATAGAAG-3', and internal primers PCAPD 5730F: 5'-ACTGGGCACAACAGACAATC-3' and PCAPD 6249R: 5'-ATATTCGGCAAGCAGGCATC-3'.

2. For detection of RNAi insert in STN-PCR reactions use external primers 35S-PDSFw: 5'-CCTAACAGAACTCGCCGTAA-3' and DirAllNco-Rv: 5'-ATGCCATGGGGTTATTGGGTGCAGAATGG-3', and internal primers Probe_5027_Fw: 5'-gtatttgtgaccatgtttctg-3' and Probe_7228_Rv: 5'-GGACGGATAGTAAACTGCGG-3'.

3. Harvest peanut pods from STN-PCR positive plants and of the control plants grown in the same conditions. CRITICAL STEP: Ensure that control plants are grown in the same conditions and the same season as the RNAi plants. Water blast the pods with a pressure washer at low intensity or scrape by hand to remove exocarp, determine the color of the mesocarp by placing the pods on a maturity board and separate pods in groups (yellow, orange, brown and black) ${ }^{35}$ (Figure 2).
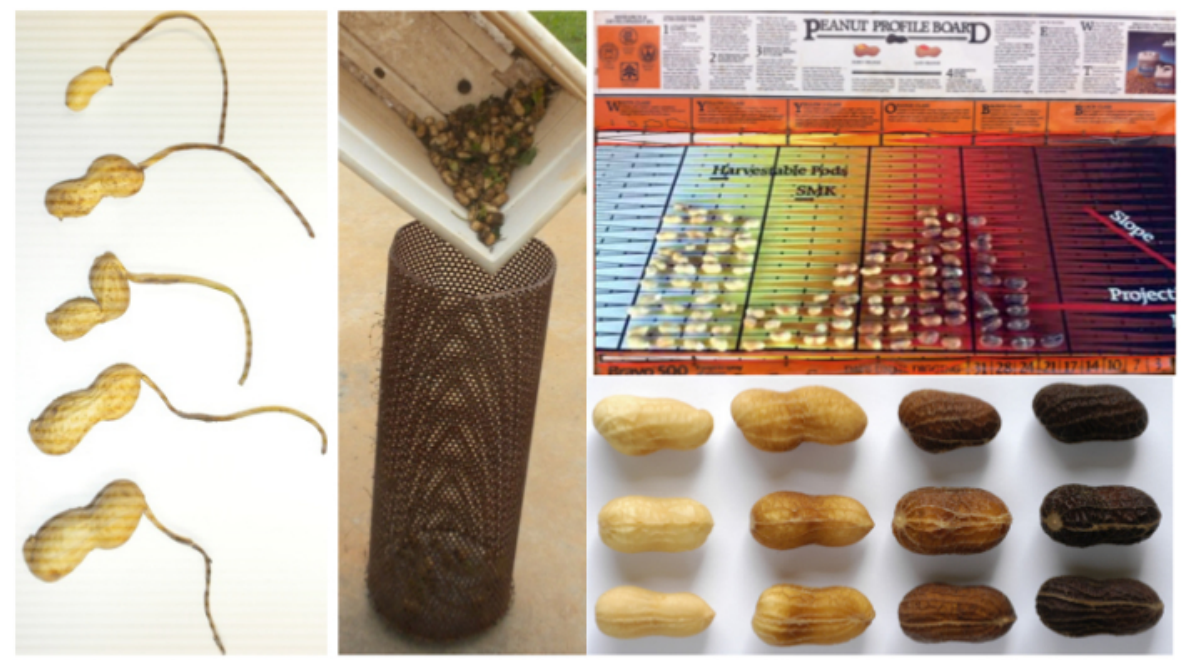

Figure 2. Preparation of peanut pods for analysis. Left: Different peanut sizes found at harvest as peanut is an indeterminate growth plant; Center: placing peanuts in metal basket for water pressure removal of exocarp; Right: maturity groups by mesocarp color on a peanut profile board (yellow, orange, brown and black). Please click here to view a larger version of this figure.

\section{Experimental Setup}

1. Remove the hulls, process yellow and brown seeds separately. Calculate the number of seeds to use in the experiment according to Table 2 . Note that a minimum of three seed pieces ( 1 seed piece $=$ half cotyledon) per peanut line and sampling date is required to perform statistical analysis and reduce the standard error. 


\begin{tabular}{|c|c|}
\hline Name & Sequence \\
\hline DIR-1 & $\begin{array}{l}\text { 5'- } \\
\text { GCCAGCTCAAAAGTGCGATGCACCAAGGAGAAACCGGCCTGTGCT } \\
\text { CGGTGTATCGAACGTGGTCTTGCCTGTCAATACATGGTCGTATTTG } \\
\text { TGACCATGTTTCTGGTGGCATTGGACCGTCTTGTCATCTCTACAGCC } \\
\text { ATTCCCCAGATCACGGACGAATCCCCTGCATCTACGCGCACGCATC } \\
\text { ACTTGGGGTACCCGT-3' }\end{array}$ \\
\hline Short-Dir1-R & 5'-Phos-TACCCCAAGTGATGCGTGCGCG-3' \\
\hline DIR-2-inverted & $\begin{array}{l}\text { 5'- GGTTATTGGGTGCAGAATGGTAAACCACCCAACAGTACGCGAAATG } \\
\text { TCAATTCCAGAGTCCCAAACCTCCCTACCGTGGCCTGGACGGATAG } \\
\text { TAAACTGCGGAGCTTGGGAACAAAATCCGCTGTCTGATCGCCGAAG } \\
\text { AGAAAGAGTTGCCTTGATTGAGCCGCATCGAGGACAGGTTGTGTTG } \\
\text { CTGTTGATAGACGGG-3' }\end{array}$ \\
\hline Short-Dir2-R & 5'-Phos-CTATCAACAGCAACACAACC-3' \\
\hline DirAll-cacc-Fw & 5'-CACCGCCAGCTCAAAAGTGCGATGC-3' \\
\hline DirAll-Nco-Rv & 5'-ATGCCATGGGGTTATTGGGTGCAGAATGG-3' \\
\hline DirAll-BamEco-Fw & 5'-ATGGGATCCGAATTCGCCAGCTCAAAAGTGCGATGC-3' \\
\hline Complete RNAi insert & $\begin{array}{l}\text { 5'- GCCAGCTCAAAAGTGCGATGCACCAAGGAGAAACCGGCCTGTGCT } \\
\text { CGGTGTATCGAACGTGGTCTTGCCTGTCAATACATGGTC/GTATTTG } \\
\text { TGACCATGTTTCTGGTGGCATTGGACCGTCTTGTCATCTCTACAGCC } \\
\text { ATTCCCCAGATCACGGACGAAT/CCCCTGCATCTACGCGCACGCAT } \\
\text { CACTTGGGGTACCCGTCTATCAACAGCAACACAACCTGTCCTCGAT } \\
\text { GCG/GCTCAATCAAGGCAACTCTTTCTCTTCGGCGATCAGACAGCG } \\
\text { GATTTTGTTCCCAAGCTCCGCAGTTTACTATCCGTCCA/GGCCACGG } \\
\text { TAGGGAGGTTTGGGACTCTGGAATTGACATTTCGCGTACTGTTGGG } \\
\text { TGGTTTACCATTCTGCACCCAATAACC-3' }\end{array}$ \\
\hline
\end{tabular}

Table 1. Oligonucleotides and ultramers used to build the RNAi construct p5XCAPD. Phos: phosphorylated 5' end; " $"$ " separates the five gene fragments used; Complete RNAi insert: sequence used as 2 inverted repeats to form p5XCAPD.

2. Place entire peanut seeds in a single layer so that they cover the bottom of a sterile beaker. Add $75 \%$ ethanol/water ( $/ \mathrm{v}$ ) solution to cover the seeds and then add an equal volume of the same solution. Incubate at ambient temperature for 30 sec and then rinse with sterile deionized water (SDW).

3. Add $2 \%$ hypochlorite to the beaker containing the ethanol treated seeds as follows: add enough $2 \%$ hypochlorite solution to cover the seeds, then add an equal volume of the same solution and incubate for 5 min. CRITICAL STEP: Rinse thoroughly three times with a volume of SDW equivalent to 5 -fold the volume of hypochlorite used (Figure 3).

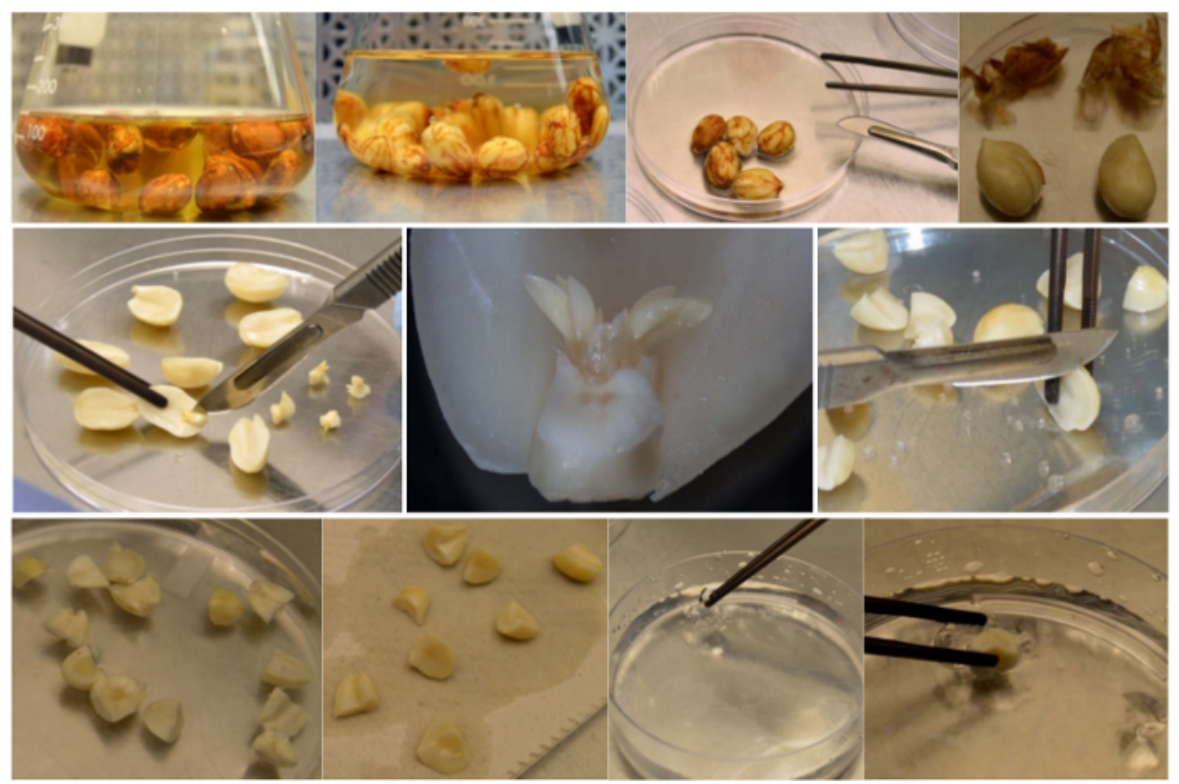

Figure 3. Experimental setup. Top: Surface sterilization of peanut seeds before and after hypochlorite, removal of seed coats (testa); Middle: removal of embryo and close view of embryo, then cut cotyledon in half; Bottom: half cotyledons in sterile distilled water, blotting on sterile absorbent paper, dents on water agar, and placing of half cotyledons (cut side upwards) on the agar surface. Please click here to view a larger version of this figure. 
4. Allow the surface-sterilized seeds to imbibe submerged in SDW for $2 \mathrm{hr}$. Place the seeds on a sterile Petri dish, remove the seed coats with forceps, separate the cotyledons, and with a scalpel remove the embryos. Note: Embryos can be discarded or used to regenerate new plants.

5. Cut each cotyledon in half using a scalpel. To avoid dehydration, keep the cut seed pieces in sterile water until all the seeds are processed.

6. Have prepared Petri dishes containing sterile water agar (1.5\% agar/water; w/v), one for each three seed pieces. Make small dents in the agar using forceps, briefly blot the excess water of seed pieces on sterile paper towels, and then place the seed pieces (cut face upwards) on the water/agar plates.

7. From a fresh culture of aflatoxigenic Aspergillus flavus NRRL 3357 in Czapek's agar medium grown at $25^{\circ} \mathrm{C}$ for 10 days, prepare a suspension of 50,000 spores per $\mu \mathrm{SDW}$, counted with a haemocytometer

8. Place $2 \mu \mathrm{l}$ of the spore suspension on the cut surfaces of each half-cotyledon piece avoiding runoff on the sides, to make sure the spores get exposed to the seed tissue that harbors RNAi (Figure 4).
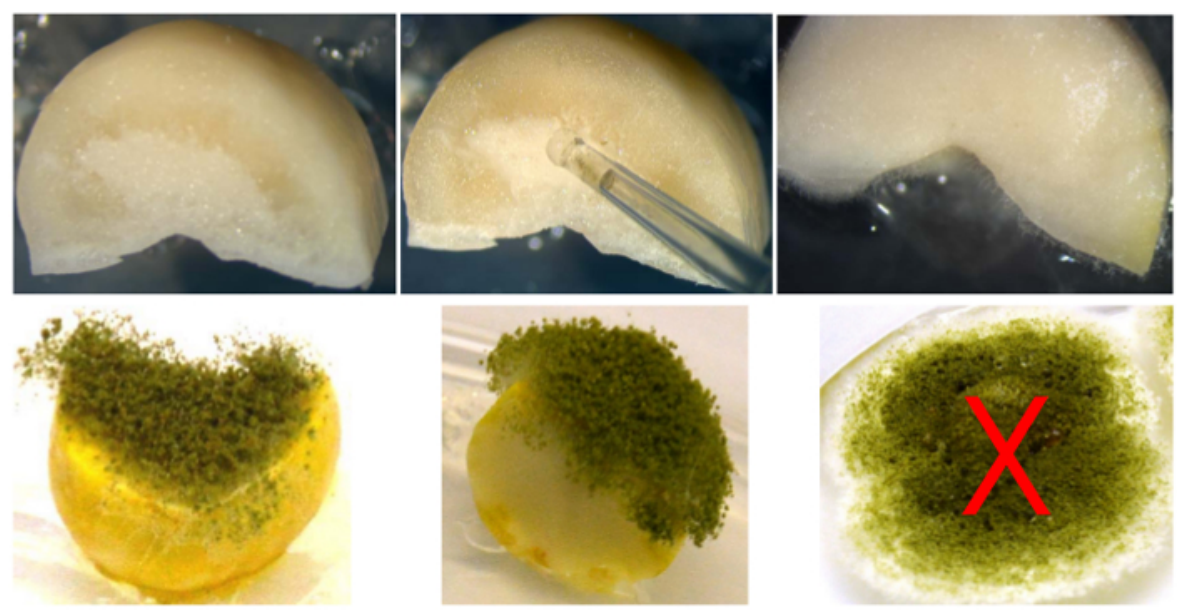

Figure 4. Inoculation and incubation for aflatoxin analysis. Top: Half cotyledon, inoculation with spore suspension, and Aspergillus flavus mycelial growth on half cotyledon after $24 \mathrm{hr}$ incubation. Bottom: left: incubation of $48 \mathrm{hr}$ on $1.5 \%$ agar; center: incubation for $72 \mathrm{hr}$ on $1.5 \%$ agar; right: example of incorrect experimental setup, incubation for $72 \mathrm{hr}$ on $0.5 \%$ agar. Please click here to view a larger version of this figure.

9. Incubate the Petri dishes containing inoculated and non-inoculated half cotyledons at $30^{\circ} \mathrm{C}$ in the dark until sampling.

\section{Sampling for Aflatoxin Analysis and Gene Expression}

1. Collect samples in triplicate at 24,48 and $72 \mathrm{hr}$ (optional $96 \mathrm{hr}$ ) of incubation, both for RT-PCR and for aflatoxin analysis, each replicate being one piece (half cotyledon). Pick samples at random from different plates on each sampling date. Using a tissue, softly remove agar and excess fungal spores before placing seed pieces in vials or test tubes.

2. For aflatoxin analysis, place each replicate (one piece) in a $4 \mathrm{ml}$ glass screw cap vial and store at $-80^{\circ} \mathrm{C}$. Note: Given the chemical stability of aflatoxins, samples can be stored in this condition for several months (in the current experiments usually 1-2 months).

3. For RT-PCR place each replicate (one piece) in already prepared $2 \mathrm{ml}$ grinding tubes containing two stainless steel beads ( $2.5 \mathrm{~mm}$ diam) and three zirconium beads $(2 \mathrm{~mm}$ diam).

4. Immediately freeze (preferably in liquid nitrogen) all the samples and keep them at $-80^{\circ} \mathrm{C}$ until processing.

\section{Aflatoxin Analysis of Individual Half Cotyledon Pieces}

1. Use the $A$. flavus inoculated samples for this analysis. Bring samples to ambient temperature for about 30 min, add four volumes (usually $2-3$ $\mathrm{ml} ; \mathrm{w} / \mathrm{v}$ ) of methanol (keep record for later calculations), close the caps, and incubate O/N ( 16 hr) in the dark without agitation.

2. Place a frit into a matching $1.5 \mathrm{ml}$ propylene minicolumn, add $200 \mathrm{mg}$ basic $\mathrm{Al}_{2} \mathrm{O}_{3}$ and cap it with another frit as described previously ${ }^{36}$; then, place an Ultra-High Performance Liquid Chomatographer (UPLC) autosampler vial under the column close enough to avoid possible evaporation of the eluate.

3. In a disposable glass test tube (do not use plastic), place $0.5 \mathrm{ml}$ of the methanol extract obtained in step 4.1 , add $0.5 \mathrm{ml}$ acetonitrile, mix by pipette and apply $0.5 \mathrm{ml}$ of the mixture into the column prepared in step 4.2 . Allow elution into the autosampler vial by gravity (do not apply pressure). Elution usually takes $2-4 \mathrm{~min}$, close the vial immediately using a UPLC compatible cap with septa. Keep the vials at ambient temperature and analyze them the same day on a UPLC.

4. For separation of aflatoxins, place autosampler vials containing sample eluates and autosampler vials with aflatoxin standards $\left(B_{1}\right.$ and $B_{2}$ when using $A$. flavus) in a UPLC instrument equipped with a matching UPLC Quaternary Solvent Manager, UPLC Sample Manager, UPLC Fluorescent Detector, and an $\mathrm{C}_{18} 2.1 \mathrm{~mm} \times 50 \mathrm{~mm}, 1.7 \mu \mathrm{m}$ column.

1. Use an isocratic mobile phase composed of water/MeOH/CH $3 \mathrm{CN}(64: 23: 13, \mathrm{v} / \mathrm{v} / \mathrm{v})$ mixture at the flow rate of $0.30 \mathrm{ml} \mathrm{min}{ }^{-1}$. Obtain chromatograms assuring a stabilized baseline separation for accurate calculation of aflatoxin concentration, according to manufacturer's instructions ${ }^{37}$.

2. Confirm the identity of aflatoxins by obtaining their mass-spectral data and comparing them with published data ${ }^{38}$. Use an ion trap mass spectrometer equipped with an ESI interface and corresponding software according to manufacturer's instructions.

5. Determine concentrations of aflatoxins by reference to calibration curves obtained by injecting different amounts of corresponding commercial standards of aflatoxins $B_{1}, B_{2}, G_{1}$ and $G_{2}$ as suggested by the UPLC manufacturer and determined by the software ${ }^{37}$. 
6. Place seed pieces already extracted with methanol, in individual glass vials for $\mathrm{O} / \mathrm{N}(\sim 16 \mathrm{hr})$ lyophilization to determine their dry weight. Then, calculate aflatoxin concentration in $\mathrm{ng} \mathrm{g}^{-1}$ of dry weight of seed piece.

7. For analysis of data, convert aflatoxin results to $\log \left(\mathrm{ng}^{-1}+1\right)$, followed by Tukey's test for mean comparisons.

\section{Gene Expression, RT-PCR Processing of Samples}

1. Take the $2 \mathrm{ml}$ grinding tubes containing samples from the $-80{ }^{\circ} \mathrm{C}$ freezer, and immediately (without thawing) grind them in a bead mill homogenizer at 3,100 rpm for $40 \mathrm{sec}$, then proceed to RNA extraction using Trizol according to manufacturer's instructions.

2. Prepare cDNA from each sample using $1 \mu \mathrm{g}$ RNA and equal amounts of oligo dT and random hexamers, make a 1:8 dilution of the cDNA and use $2 \mu$ l per reaction in RT-PCR (as previously described ${ }^{39}$ ).

1. For detection of expression of RNAi insert use primers: RT_5X_1_105F: 5'GGTGGCATTGGACCGTCTTG-3', RT_5X_1_232R: 5'-CGCATCGAGGACAGGTTGTG-3'; and RT_5X_2_95F: 5'-CC̄ATGTTTCTGGTGGCATTG-3', RT_5X 2 229R: 5'ATCGAGGACAGGTTGTGTTG-3'.

2. For detection of expression of the selectable marker NPTII, use primers: RT_NPTII_1_6871F: 5'-CTCGCTCGATGCGATGTTTC-3', RT_NPTII_1_7004R: 5'-GCAGGATCTCCTGTCATCTC-3'. Use the housekeeping gene Actin for standardization, and primers: ActinFw: CACATGCCATCCTTCGATTG; Actin-Rv: CCAAGGCAACATATGCAAGCT ${ }^{40}$.

3. Analyze results by Delta-delta $\mathrm{C}_{\mathrm{T}}$ method ${ }^{41}$ standardized for Actin expression. Represent the results as fold increase over the control.

\section{Representative Results}

Plasmid p5XCAPD was made as a derivative of pCAPD ${ }^{29}$, and used it to transform peanut plants; this vector carries inverted repeats of five small fragments, 70-80 bp each, of aflatoxin-synthesis genes of $A$. flavus separated by an intron (Figure 1). Fragments of AFL2G_07224 (aflR), AFL2G_07223 (aflS or aflJ), AFL2G_05027 (aflatoxin efflux pump, aflep), AFL2G_07228 (aflC/pksA/pksL1), and AFL2G_07731 (pes1) were used for the construct, numbers on Figure 1 correspond to $A$. flavus genome annotation in BROAD Institute, Cambridge, MA, and the literature ${ }^{42}$. A total of 99 peanut lines were regenerated after going through the transformation process, 50 were PCR positive for NPTII detected by STNPCR, and 33 lines were PCR positive and produced seeds. Only seven PCR positive lines were clonally propagated and tested by the present method for aflatoxin accumulation, all seven showed between $60 \%$ and $100 \%$ less aflatoxin accumulation than the control. Here we show results of two of those seven lines. As individual transgenic events usually produce few seeds, a method was developed to use a minimum number of seeds while still being able to do parametric statistical analysis. A flowchart of the sample preparation and experimental setup is shown in Figure 5 and Table 1. Though the first generation of transgenic seeds is typically hemizygous, it is expected that the cell-to-cell and systemic movement of small interfering RNA (siRNA) generated through RNA interference should confer aflatoxin-synthesis silencing throughout the plant.

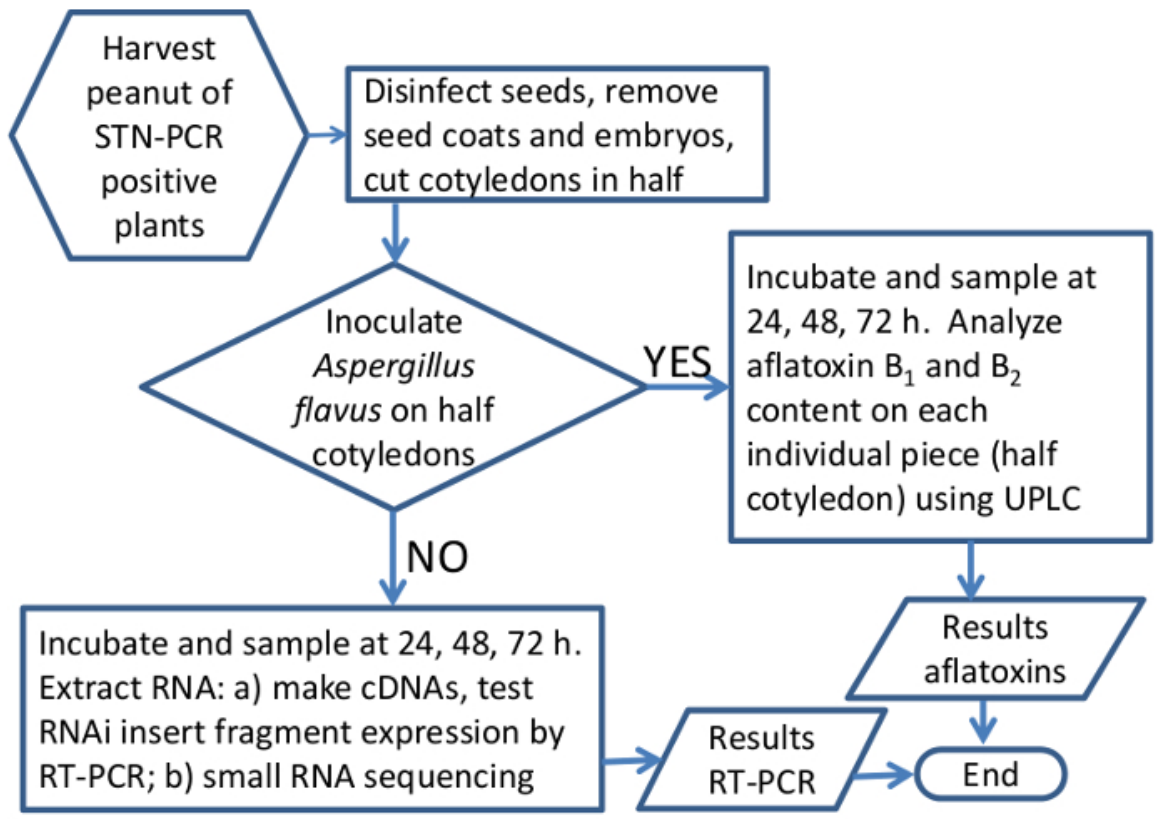

Figure 5. Schematic flowchart of the method to analyze effectiveness of RNAi in silencing Aspergillus aflatoxin-synthesis genes in peanut seeds. Graphic representation of the work flow when processing peanut samples for gene expression or aflatoxin analysis. Please click here to view a larger version of this figure.

RNAi peanut lines 288-72 and 288-74 showed presence of the NPTIl selectable maker when tested by STN-PCR, gel sections are shown in Figure 6 (top), original pictures are available from the authors upon request. Plasmid pCAPD was not used as a control of transformation since it encodes inverted repeats of two genes $\mathrm{Cmr}$ (chloramphenicol resistance) and $\mathrm{CcdB}$ (toxin) of unknown effect on plants. PCR negative peanut line 288-9 and others which went through the regeneration process and was grown in the same conditions as RNAi lines, were used as negative control. 


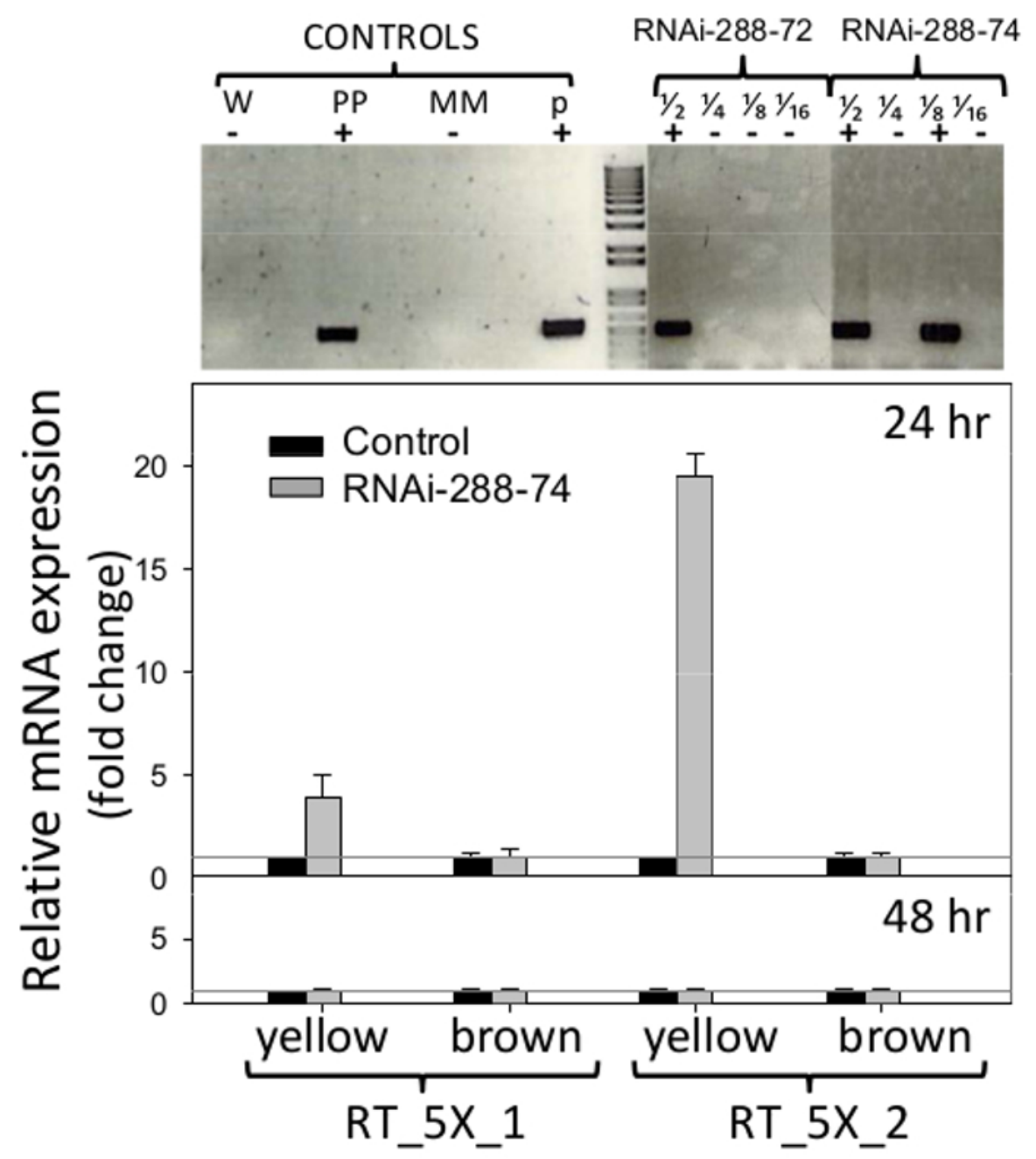

Figure 6. Detection of transgenics and Real Time expression of RNAi insert. Top: Single-tube, Nested PCR detection of transgenic peanut lines RNAi-288-72 and RNAi-288-74, positive plants. Controls: W (water), PP (positive plant), MM (master mix), p (plasmid p5XCAPD); fractions $1 / 2 \frac{1}{4} 1 / 81 / 16$ represent 2 -fold dilutions of DNA. Bottom: Real-Time PCR detection of expression of the RNAi insert (primer sets: RT_5X_1, RT_5X_2, as in Figure 1, on immature (yellow) and mature (brown) cotyledons of transgenic lines at 24 and $48 \mathrm{hr}$ incubation; gray line: $\bar{C}_{T}$ $=1$. Histograms represent the means and standard error bars $(T)$ of three biological samples with three technical replicates. The relative quantification of RNAi insert was normalized with respect to the housekeeping gene Actin as an internal control and comparative fold expression of the transgene calculated as described in 5.2.2 and 5.3. Please click here to view a larger version of this figure.

To test the effectiveness of plant-host RNAi-mediated potential control of aflatoxin accumulation, freshly harvested conidia of Aspergillus flavus NRRL 3357 were applied on the cut surface of half cotyledons from which the embryos and testa had been removed (Figures 3,4$)$. A. flavus NRRL 3357, for which the genome has been sequenced and was the basis for designing p5XCAPD, was kindly provided by Dr. Horn at USDAARS-NPRL. The resulting fungal invasion of half cotyledon after 24,48 and $72 \mathrm{hr}$ at $30^{\circ} \mathrm{C}$ is shown in Figure 4 . Samples of inoculated half cotyledons were collected at $24,48,72,96 \mathrm{hr}$ of incubation, and analyzed for the main four aflatoxins $B_{1}, B_{2}, G_{1}$ and $G_{2}$ using UPLC and confirmed by LC-MS; results are shown in Figure 6. Aflatoxin concentrations were determined using a published method with modifications 36. At $96 \mathrm{hr}$ incubation, cotyledons start to disintegrate because of the fungal infection. RNAi line 288-72 exhibited significantly lower levels of aflatoxins than the control at all sampling dates in immature cotyledons and at most sampling dates in the mature ones. RNAi line 288-74 showed significantly lower levels of aflatoxins at most sampling dates. Levels of significance of Tukey's test are indicated with asterisks in the graphic of Figure 7. 

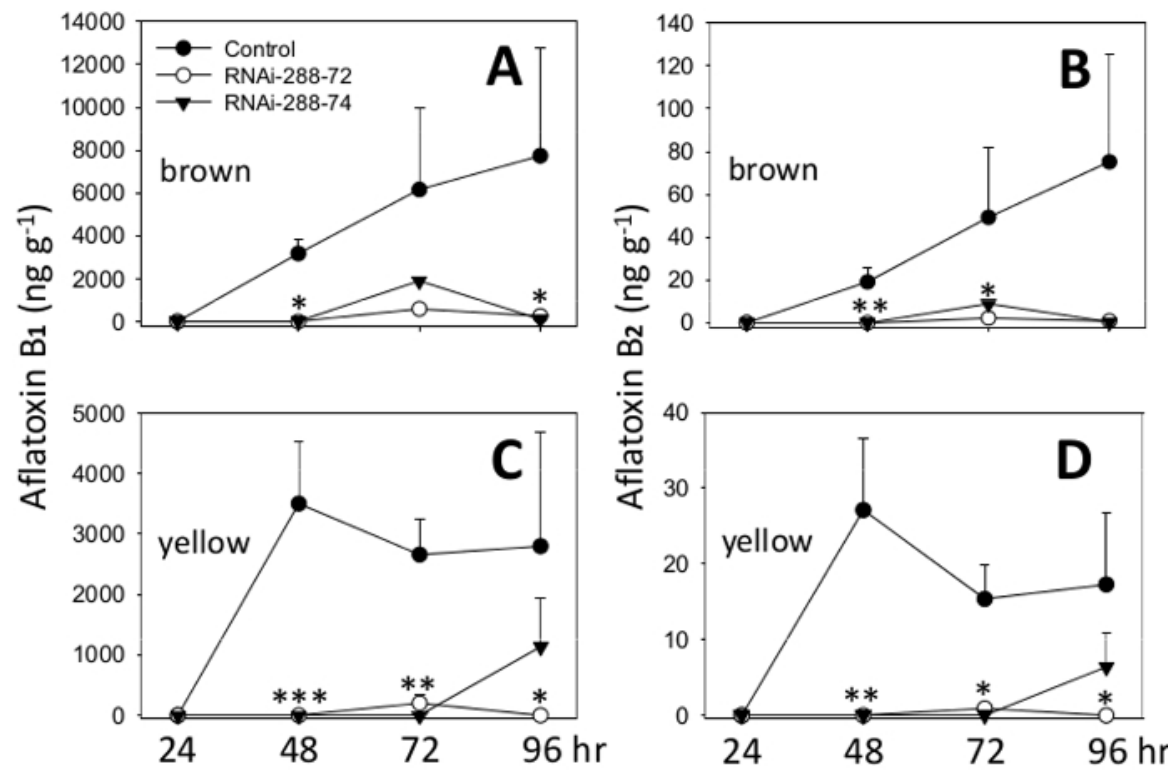

Figure 7. Aflatoxins $B_{1}$ and $B_{2}$ in half peanut cotyledons after incubation $(\mathbf{2 4}, \mathbf{4 8}, \mathbf{7 2}$ and $\mathbf{9 6} \mathrm{hr})$ with Aspergillus flavus. Control: seeds of 288-9 non-transgenic line; RNAi: seeds from RNAi-288-72 and RNAi-288-74, transgenic for RNAi p5XCAPD to silence five aflatoxin-synthesis genes. (A) Aflatoxin $B_{1}$ in mature seeds (brown); (B) Aflatoxin $B_{2}$ mature seeds; (C) Aflatoxin $B_{1}$ in immature seeds (yellow) and (D) Aflatoxin $B_{2}$ in immature seeds. Mean values with the corresponding standard error bars $(T)$ of duplicate biological samples are represented. Statistically significant differences Tukey's test ${ }^{*}: p \leq 0.05,{ }^{* *}: p \leq 0.01,{ }^{* * *}: p \leq 0.001$. Please click here to view a larger version of this figure.

Overall, RNAi-288-72 showed throughout the experiment (24 to $96 \mathrm{hr}$ incubation), a $94 \%-100 \%$ reduction in aflatoxin $\mathrm{B}_{2}$, and a $90 \%-100 \%$ reduction in aflatoxin $B_{1}$ compared to the control. RNAi-288-74 showed a $63 \%-100 \%$ reduction in aflatoxin $B_{2}$ and $60 \%-100 \%$ reduction in aflatoxin $\mathrm{B}_{1}$, Figure 7 .

Primers used for Real-Time PCR detection of expression of the RNAi insert are shown in Figure 1. Peanut-seed cotyledons were analyzed without embryos, to remove their natural defenses and be able to detect the potential effect of RNAi on the area most exposed to fungal invasion, the cotyledons. Expression of the RNAi insert detected in immature cotyledons (yellow) of line 288-74 by primer set RT_5X_1 was four fold over the $\mathrm{C}_{\mathrm{T}}=1$ threshold of the negative control, and by primer set RT_5X_2 was 19 fold above the threshold, all at $24 \mathrm{hr}$ incubation. At least three out of five consecutive gene fragments used in the peanut transformation, 5027, 7223 and 7228 (aflep, aflS/aflJ, and aflC/pksA, respectively) were detected by RT-PCR (Figure 1, 6). Expression of RNAi insert was not detected in mature cotyledons at $24 \mathrm{hr}$, or on mature or immature cotyledons at $48 \mathrm{hr}$ incubation, Figure 6 (bottom).

\begin{tabular}{|c|c|c|c|c|c|c|c|c|}
\hline \multirow[t]{3}{*}{ Peanut Line } & \multirow{3}{*}{$\begin{array}{l}\text { Time of } \\
\text { sampling }\end{array}$} & \multicolumn{3}{|c|}{ Samples for RT-PCR } & \multirow{2}{*}{\multicolumn{3}{|c|}{ Samples for aflatoxin analysis (inoculated) }} & \multirow{3}{*}{$\begin{array}{l}\text { Number of } \\
\text { seeds }\end{array}$} \\
\hline & & \multicolumn{3}{|c|}{ (non inoculated) } & & & & \\
\hline & & $\operatorname{Rep} 1$ & $\operatorname{Rep} 2$ & $\operatorname{Rep} 3$ & Rep 1 & $\operatorname{Rep} 2$ & Rep 3 & \\
\hline \multirow[t]{3}{*}{ RNAi (yellow) } & $24 \mathrm{hr}$ & 1 & 1 & 1 & 1 & 1 & 1 & \multirow[t]{3}{*}{4.5} \\
\hline & $48 \mathrm{hr}$ & 1 & 1 & 1 & 1 & 1 & 1 & \\
\hline & $72 \mathrm{hr}$ & 1 & 1 & 1 & 1 & 1 & 1 & \\
\hline Control & $24 \mathrm{hr}$ & 1 & 1 & 1 & 1 & 1 & 1 & \multirow[t]{3}{*}{4.5} \\
\hline \multirow[t]{2}{*}{ (yellow) } & $48 \mathrm{hr}$ & 1 & 1 & 1 & 1 & 1 & 1 & \\
\hline & $72 \mathrm{hr}$ & 1 & 1 & 1 & 1 & 1 & 1 & \\
\hline
\end{tabular}

Table 2. Example of small-sample setup to analyze gene expression and aflatoxin accumulation in RNAi peanut seeds. Complete analysis of expression and aflatoxins for one maturity group (i.e., yellow), with three sampling times (24, 48 and $72 \mathrm{hr}$ ), in triplicate, would require 4.5 seeds, each number one in the table represents half cotyledon.

\section{Discussion}

Plant-host RNAi-mediated silencing of genes in fungal pathogens has been demonstrated ${ }^{27,43}$, however, there are no publications showing the feasibility of RNAi-mediated control of mycotoxin accumulation in plants. One limiting factor for these studies in peanut was the lack of a method to evaluate a no-aflatoxin accumulation phenotype in individual plants, as leaves show no symptoms upon fungal infection of underground pods. In addition, the not-normally distributed accumulation of aflatoxins, and the need for large samples for chemical analysis ${ }^{15,16}$ have hindered the quantification of potential RNAi effect on a single plant. The method presented here consists of $72 \mathrm{hr}$ experiments using five seeds to perform 
three $24 \mathrm{hr}$-interval samplings in triplicate (Table 1, Figure 7). Compared to the typical aflatoxin analysis that requires no less than $100 \mathrm{~g}$ of seeds, our method is particularly suitable for individual transgenic events of peanut plants which initially produce no more than two or three pods.

RNA-mediated silencing of aflatoxin synthesis has been demonstrated by genetically transforming Aspergillus flavus and $A$. parasiticus. Since afIR is a main regulator of aflatoxin production in $A$. flavus and $A$. parasiticus ${ }^{44,45}$, it becomes an interesting target for RNA-mediated silencing in plants. However, genetic variations in afIR have been shown among Aspergillus species ${ }^{46}$, and those genetic variants could escape silencing if there is no perfect sequence matching with the RNAi signal produced in the plant host. Thus, aflR was one of the targets for silencing in vector p5XCAPD, but was not the only one. Inverted repeats of the afIR gene introduced into A. flavus and $A$. parasiticus by transformation resulted in silencing and minimal or no production of aflatoxins ${ }^{47}$ (McDonald et al., 2005b). Also, silencing afID gene prevented aflatoxin production by up to $98 \%$ in $A$. flavus and $A$. parasiticus in direct transformation ${ }^{48}$. To increase the probability of success in our system, peanut was transformed with inverted repeat fragments of five genes involved in aflatoxin production in $A$. flavus. Here it is shown that using p5XCAPD that targets for silencing several genes in the aflatoxin synthesis pathway, $90 \%-100 \%$ lower levels of aflatoxin $B_{1}$ and $B_{2}$ were achieved in line $288-72$, and $60-100 \%$ lower levels accumulated in line 288-74 compared to the control, when half cotyledons were inoculated with $A$. flavus, Figures 4 , 7. Most importantly, this method detected statistically significant differences in aflatoxin accumulation by lines 288-72, 288-74 vs. the control throughout the experiment by applying parametric statistics, Figure 7. Given the small sample size, it is important to highlight the need for using a powerful method to detect aflatoxins, these experiments were analyzed by UPLC which has a high resolution, five-fold higher performance and three times higher sensitivity than HPLC ${ }^{49}$.

Expression of the RNAi insert in 288-74 was only detected in immature cotyledons (yellow) at $24 \mathrm{hr}$ incubation. The RNAi insert was not detected by RT-PCR on mature cotyledons of 288-74 at $24 \mathrm{hr}$, or on any maturity group at $48 \mathrm{hr}$, Figure 6 . This same phenomenon was observed in other RNAi transgenic peanut lines (Arias, R.S., 2015 unpublished), where usually RNAi transcripts were only detected on immature cotyledons at $24 \mathrm{hr}$. RNA samples were treated with DNAse before cDNA synthesis, data were normalized to the level of Actin expression and no evidence of DNA contamination was observed. Should DNA have been present in the samples, it should have been detected in the $48 \mathrm{hr}$ samples as well, but consistently that was not the case. Expression under the control of the 35S-promoter is not always uniform; it can be affected by environmental conditions ${ }^{50}$, type of tissue and developmental stage ${ }^{51,52}$. At the same time, in the pathway of RNA interference, the rate of mRNA decay and the rate of siRNA decay can vary significantly ${ }^{53}$. It is possible that the rapid degradation of the mRNA by the mechanism of RNA interference could have prevented mRNA detection at $48 \mathrm{hr}$ incubation. Whether absence of expression at $48 \mathrm{hr}$ was due to low 35S-promoter driven transcription, or to fast degradation of dsRNA by Dicer remains to be answered. Thus, detection of small RNAs by high throughput sequencing would give a better insight on the processes taking place through RNAi ${ }^{54}$ in these experiments. However, since RNA silencing spreads systemically, mainly through the phloem from photosynthate sources to sucrose sinks (in this case peanut seeds) ${ }^{55}$, the silencing of aflatoxin-synthesis can occur in seeds without local expression of the RNAi insert. Much research remains to be done to determine the threshold level of small interfering RNAs (siRNAs) necessary to prevent aflatoxin accumulation in seeds. It is important to emphasize the fact that both, mRNA expression of the RNAi construct (Figure 6), and accumulation of aflatoxins $B_{1}$ and $B_{2}$ (Figure 7) showed different results for immature (yellow) vs. mature (brown) cotyledons. Peanut plants have indeterminate growth, that is, they present at harvest a range of maturity pods, Figure 2. In addition, seeds from different maturity groups differ in their chemical composition, e.g., $2.4 \%$ sucrose in immature seeds, and $1.9 \%$ in mature seeds under the same field conditions ${ }^{56,57}$. Thus, to understand the actual efficiency of RNA-mediated control of aflatoxin accumulation, it is important to analyze maturity groups separately.

A natural defense of peanut seeds is the production of phytoalexins, which varies in the diversity of compounds produced and their relative quantities depending on the maturity of the seeds and environmental conditions ${ }^{58-61}$, and it is particularly higher in embryos compared to cotyledons ${ }^{62}$. Embryos also have significantly higher concentrations of nucleic acids, both DNA and RNA than the cotyledons (Arias R.S., unpublished). As peanut seeds mature, changes in their physiology and chemical composition occur ${ }^{63}$. Phenolic antioxidants in peanut testa form condensed tannins with fungistatic activity ${ }^{64}$; this is also evident in the mesocarp color that reflects maturity stages, yellow to black ${ }^{35}$, as its content of tannins and phenolic compounds increases with maturity ${ }^{65}$. Thus, presence of testa or embryos in the experiment, given their antimicrobial properties, could have limited fungal growth and therefore overestimated the effect of RNAi silencing, therefore, they were removed. Also, removal of testa and embryos helps limit the sources of variation in the analysis, as the half cotyledon that carries the embryo will have more phytoalexins and more RNA content.

In addition to the analysis by maturity groups and removal of testa and embryo in these experiments, it is important to point out few more observations: a) though results are shown for up to $96 \mathrm{hr}$ incubation, it is recommended to use no more than 72 hr to obtain consistent results, as seeds get degraded by $96 \mathrm{hr}$; and b) whereas half cotyledons from the same seed, though sampled at random, do not constitute perfectly independent samples, RT-PCR and aflatoxin accumulation within transgenic events showed minimum variation between seeds. Also, an accurate fungal spore count, inoculum volumes of $2 \mu \mathrm{l}$, and application of spores on the cut surface of the cotyledons avoiding dripping on the sides are important to make sure the germinated spores are exposed to the plant tissue. The water/agar on the plates should be at $1.5 \%$ ( $\mathrm{w} /$ v), softer agar causes runoff of spores as shown on the last frame of Figure $\mathbf{4}$ (bottom). Should seed availability from a particular transgenic event be limited, sampling can be done in duplicate instead of triplicate obtaining similar results (i.e., Figure 7); however, triplicate samples will help reduce the standard error. The only limitation of this method is that it requires a highly sensitive system (UPLC) for aflatoxin detection/ quantification, but at the same time this reduces the probability of overestimating the effect of RNAi should aflatoxins not be detected by less sensitive methods.

In conclusion, this method offers for the first time a reliable approach to study the effect of RNAi in the control of aflatoxins. Reducing the time for an experiment from an entire cropping season to less than one week, this method will tremendously accelerate the research on RNAipeanut/Aspergillus pathosystem towards the mitigation and/or elimination of aflatoxins.

\section{Disclosures}

The authors have nothing to disclose or any conflicts of interest. 


\section{Acknowledgements}

This work received the financial support of USDA-ARS CRIS project 6604-21000-004-00D, CRIS project 6604-42000-008-00D, and USAID Feed-the-Future program Agreement number 58-0210-3-012. We thank Valerie Orner, LaTanya Johnson, Joseph Powell and Kathy Gray for their technical assistance. Mention of trade names or commercial products in this article is solely for the purpose of providing specific information and does not imply recommendation or endorsement by the US Department of Agriculture.

\section{References}

1. Williams, J. H. et al. Human aflatoxicosis in developing countries: a review of toxicology, exposure, potential health consequences, and interventions. The American Journal of Clinical Nutrition. 80, 1106-1122 (2004).

2. American Association for Cancer Research: AACR. An evaluation of chemicals and industrial processes associated with cancer in humans based on human and animal data: IARC Monographs Volumes 1 to 20. Cancer Research. 40, 1-12 (1980).

3. Turner, P. C. The molecular epidemiology of chronic aflatoxin driven impaired child growth. Scientifica. 2013 (2013).

4. Rasooly, R., Hernlem, B., He, X., \& Friedman, M. Non-linear relationships between aflatoxin $B_{1}$ levels and the biological response of monkey kidney vero cells. Toxins (Basel). 5, 1447-1461 (2013).

5. Gong, Y. Y. et al. Determinants of aflatoxin exposure in young children from Benin and Togo, West Africa: the critical role of weaning International Journal of Epidemiology. 32, 556-562 (2003).

6. Eaton, D. L., \& Groopman, J. D. The toxicology of aflatoxins: human health, veterinary, and agricultural significance. Academic Press, (1994).

7. Murugavel, K. G. et al. Prevalence of aflatoxin $B_{1}$ in liver biopsies of proven hepatocellular carcinoma in India determined by an in-house immunoperoxidase test. Journal of Medical Microbiology. 56, 1455-1459 (2007).

8. Wang, J. S. et al. Hepatocellular carcinoma and aflatoxin exposure in Zhuqing Village, Fusui County, People's Republic of China. Cancer Epidemiology, Biomarkers \& Prevention: a publication of the American Association for Cancer Research, cosponsored by the American Society of Preventive Oncology. 10, 143-146 (2001).

9. Azziz-Baumgartner, E. et al. Case-control study of an acute aflatoxicosis outbreak, Kenya, 2004. Environmental Health Perspectives. 113, 1779-1783 (2005).

10. Lye, M. S., Ghazali, A. A., Mohan, J., Alwin, N., \& Nair, R. C. An outbreak of acute hepatic encephalopathy due to severe aflatoxicosis in Malaysia. American Journal of Tropical Medicine and Hygiene. 53, 68-72 (1995).

11. Villers, P. Aflatoxins and safe storage. Frontiers in Microbiology. 5, 158 (2014).

12. Kensler, T. W., Roebuck, B. D., Wogan, G. N., \& Groopman, J. D. Aflatoxin: A 50-year odyssey of mechanistic and translational toxicology. Toxicological Sciences. 120, S28-S48 (2011).

13. Dorner, J. W., Cole, R. J., \& Wicklow, D. T. Aflatoxin reduction in corn through field application of competitive fungi. Journal of Food Protection. 62, 650-656 (1999).

14. Cotty, P. J., \& Bhatnagar, D. Variability among atoxigenic Aspergillus flavus strains in ability to prevent aflatoxin contamination and production of aflatoxin biosynthetic-pathway enzymes. Applied and Environmental Microbiology. 60, 2248-2251 (1994).

15. Whitaker, T. B. Standardisation of mycotoxin sampling procedures: an urgent necessity. Food Control. 14, $233-237$ (2003).

16. Whitaker, T. B., Dorner, J. W., Giesbrecht, F. G., \& Slate, A. B. Variability among aflatoxin test results on runner peanuts harvested from small field plots. Peanut Science. 31, 59-63 (2004).

17. Fire, A. et al. Potent and specific genetic interference by double-stranded RNA in Caenorhabditis elegans. Nature. 391, 806-811 (1998).

18. Rafael, D. et al. EMT blockage strategies: Targeting Akt dependent mechanisms for breast cancer metastatic behaviour modulation. Current Gene Therapy. (2015).

19. Li, G., Chang, H., Zhai, Y. P., \& Xu, W. Targeted silencing of inhibitors of apoptosis proteins with siRNAs: a potential anti-cancer strategy for hepatocellular carcinoma. Asian Pacific Journal of Cancer Prevention: APJCP. 14, 4943-4952 (2013).

20. Koldehoff, M. Targeting bcr-ab/ transcripts with siRNAs in an imatinib-resistant chronic myeloid leukemia patient: challenges and future directions. Methods in Molecular Biology. 1218, 277-292 (2015).

21. Zhang, J. et al. Pest control. Full crop protection from an insect pest by expression of long double-stranded RNAs in plastids. Science. 347, 991-994 (2015).

22. Ajjappala, H., Chung, H. Y., Sim, J. S., Choi, I., \& Hahn, B. S. Disruption of prefoldin-2 protein synthesis in root-knot nematodes via hostmediated gene silencing efficiently reduces nematode numbers and thus protects plants. Planta. 241, 773-787 (2015).

23. Jose, A. M., \& Hunter, C. P. Transport of sequence-specific RNA interference information between cells. Annual Review of Genetics. 41, 305-330 (2007).

24. Vazquez, F., \& Hohn, T. Biogenesis and biological activity of secondary siRNAs in plants. Scientifica. Hindawi Publishing Corporation. (2013).

25. Tinoco, M. L. P., Dias, B. B. A., Dall'Astta, R. C., Pamphile, J. A., \& Aragao, F. J. L. In vivo trans-specific gene silencing in fungal cells by in planta expression of a double-stranded RNA. BMC Biology. 8, 27 (2010).

26. Govindarajulu, M., Epstein, L., Wroblewski, T., \& Michelmore, R. W. Host-induced gene silencing inhibits the biotrophic pathogen causing downy mildew of lettuce. Plant Biotechnology Journal. (2014).

27. Yin, C., Jurgenson, J. E., \& Hulbert, S. H. Development of a host-induced RNAi system in the wheat stripe rust fungus Puccinia striiformis f. sp. tritici. Molecular Plant-Microbe Interactions. 24, 554-561 (2011).

28. Ghag, S. B., Shekhawat, U. K., \& Ganapathi, T. R. Host-induced post-transcriptional hairpin RNA-mediated gene silencing of vital fungal genes confers efficient resistance against Fusarium wilt in banana. Plant Biotechnology Journal. 12, 541-553 (2014).

29. Filichkin, S. A. et al. Efficiency of gene silencing repeats vs. transitive RNAi in Arabidopsis: direct inverted vectors. Plant Biotechnology Journal. 5, 615-626 (2007).

30. Sciaky, D., Montoya, A.L., \& Chilton, M.D. Fingerprints of Agrobacterium Ti Plasmids. Plasmid. 1, $238-253$ (1978).

31. Clark, D.J., \& Maaloe, O. DNA Replication and Division Cycle in Escherichia coli. Journal of Molecular Biology. 23, 99-112 (1967).

32. Murashige, T., \& Skoog, F. A revised medium for rapid growth and bio assays with tobacco tissue cultures. Physiol Plantarum. 15, 473-497, (1962). 
33. Srinivasan, T., Kumar, K.R.R., \& Kirti, P.B. (2010) Establishment of efficient and rapid regeneration system for some diploid wild species of Arachis. Plant Cell Tissue and Organ Culture. 101, 303-309 (2010).

34. Gomes, A. L. V. et al. Single-tube nested PCR using immobilized internal primers for the identification of dengue virus serotypes. Journal of Virology Methods. 145, 76-79 (2007).

35. Williams, E. J., \& Drexler, J. S. A non-destructive method for determining peanut pod maturity. Peanut Science. 8, 134-141 (1981).

36. Sobolev, V. S., \& Dorner, J. W. Cleanup procedure for determination of aflatoxins in major agricultural commodities by liquid chromatography. Journal of AOAC International. 85, 642-645 (2002).

37. Empower Software, Getting Started Guide. Waters Corporation, Milford, MA. http://sites.chem.colostate.edu/diverdi/C431/experiments/high \%20pressure\%20liquid\%20chromatography/references/Empower\%20getting\%20started\%2071500031203rA.pdf (2002).

38. Biselli, S., Hartig, L., Wegner, H., \& Hummert, C. Analysis of Fusarium. toxins using LC-MS-MS: Application to various food and feed matrices. LC GC North America. 23, 404-413 (2005).

39. Arias, R. S., Sobolev, V. S., Orner, V. A., Dang, P. M., \& Lamb, M. C. Potential involvement of Aspergillus flavus laccases in peanut invasion at low water potential. Plant Pathology. 63, 353-363, (2014).

40. Dang, P. M., Chen, C. Y., \& Holbrook, C. C. Evaluation of five peanut (Arachis hypogaea) genotypes to identify drought responsive mechanisms utilising candidate-gene approach. Functional Plant Biology. 40, 1323-1333 (2013).

41. Schmittgen, T. D., \& Livak, K. J. Analyzing real-time PCR data by the comparative C-T method. Nature Protocols. 3, 1101-1108 (2008)

42. Amaike, S., \& Keller, N. P. Aspergillus flavus. Annual Review of Phytopathology, 49, 107-133 (2011).

43. Nowara, D. et al. HIGS: Host-induced gene silencing in the obligate biotrophic fungal pathogen Blumeria graminis. Plant Cell. 22, $3130-3141$ (2010).

44. Woloshuk, C. P. et al. Molecular characterization of aflR, a regulatory locus for aflatoxin biosynthesis. Applied and Environmental Microbiology. 60, 2408-2414 (1994).

45. Price, M. S. et al. The aflatoxin pathway regulator AfIR induces gene transcription inside and outside of the aflatoxin biosynthetic cluster. FEMS Microbiology Letters. 255, 275-279, (2006).

46. Ehrlich, K. C., Montalbano, B. G., \& Cotty, P. J. Sequence comparison of afIR from different Aspergillus. species provides evidence for variability in regulation of aflatoxin production. Fungal Genetics and Biology. 38, 63-74 (2003).

47. McDonald, T., Brown, D., Keller, N. P., \& Hammond, T. M. RNA silencing of mycotoxin production in Aspergillus and Fusarium species. Molecular Plant Microbe Interactions. 18, 539-545 (2005).

48. Abdel-Hadi, A. M., Caley, D. P., Carter, D. R., \& Magan, N. Control of aflatoxin production of Aspergillus flavus. and Aspergillus parasiticus. using RNA silencing technology by targeting afID (nor-1) gene. Toxins (Basel). 3, 647-659 (2011).

49. Swartz, M. E. Ultra performance liquid chromatography (UPLC): An introduction. Separation Science Redefined. LCGC North America. May 2005, Supplement 8. http://www.spectroscopyonline.com/spectroscopy/data/articlestandard/lcgc/242005/164646/article.pdf, 8-14 (2005).

50. Maghuly, F., Khan, M.A., Fernandez, E.B., Druart, P., Watillon, B., \& Laimer, M. Stress regulated expression of the GUS-marker gene (uidA) under the control of plant calmodulin and viral $35 \mathrm{~S}$ promoters in a model fruit tree rootstock: Prunus incisa $x$ serrula. Journal of Biotechnology. $135,105-116(2008)$

51. de Mesa, M.C., Santiago-Doménech, N., Pliego-Alfaro, F., Quesada, M.A., \& Mercado, J.A. The CaMV 35 S promoter is highly active on floral organs and pollen of transgenic strawberry plants. Plant Cell Reports. 23, 32-38, (2004).

52. Sunilkumar, G., Mohr, L., Lopata-Finch, E., Emani, C., \& Rathore, K.S. Developmental and tissue-specific expression of CaMV $35 \mathrm{~S}$ promoter in cotton as revealed by GFP. Plant Molecular Biology. 50, 463-474 (2002).

53. Groenenboom, M. A. C., Maree, A. F. M., \& Hogeweg, P. The RNA silencing pathway: The bits and pieces that matter. PLoS Computational Biology. 1, 155-165 (2005).

54. Zhao, D., \& Song, G. Q. High-throughput sequencing as an effective approach in profiling small RNAs derived from a hairpin RNA expression vector in woody plants. Plant Science: an International Journal of Experimental Plant Biology. 228, 39-47 (2014).

55. Kamthan, A., Chauduri, A., Kamthan, M., \& Datta, A. Small RNAs in plants: recent development and application for crop improvement. Frontiers in Plant Science. 6, 208 (2015).

56. Manda, A., Bodapati, P.N., Rachaputi, N.C., Wright, G. and Fukai, S. (2004). Aflatoxins and their relationship with sugars in peanut (Arachis hypogaea L.). in 4th International Crop Science Congress.([http//www.cropscience.org.au/icsc2004/poster/5/1/3/625_manda.htm]). (2004).

57. Uppala, S. S. Factors affecting pre-harvest aflatoxin contamination of peanut (Arachis hypogaea L). Ph.D. thesis, Auburn University, (2011).

58. Sobolev, V. S. Localized production of phytoalexins by peanut (Arachis hypogaea) kernels in response to invasion by Aspergillus species. Journal of Agricultural and Food Chemistry. 56, 1949-1954 (2008).

59. Sobolev, V. S., Guo, B. Z., Holbrook, C. C., \& Lynch, R. E. Interrelationship of phytoalexin production and disease resistance in selected peanut genotypes. Journal of Agricultural and Food Chemistry. 55, 2195-2200 (2007).

60. Sobolev, V. S., Neff, S. A., \& Gloer, J. B. New stilbenoids from peanut (Arachis hypogaea) seeds challenged by an Aspergillus caelatus strain. Journal of Agricultural and Food Chemistry. 57, 62-68 (2009).

61. Dorner, J. W., Cole, R. J., Sanders, T. H., \& Blankenship, P. D. Interrelationship of kernel water activity, soil temperature, maturity, and phytoalexin production in preharvest aflatoxin contamination of drought-stressed peanuts. Mycopathologia. 105, 117-128 (1989).

62. Sobolev, V. S. Production of phytoalexins in peanut (Arachis hypogaea) seed elicited by selected microorganisms. Journal of Agricultural and Food Chemistry. 61, 1850-1858 (2013).

63. Basha, S. M. M., Cherry, J. P., \& Young, C. T. Changes in free amino acids, carbohydrates, and proteins of maturing seeds from various peanut (Arachis hypogaea L.) cultivars. Cereal Chemistry. 53, 586-596 (1976).

64. Lansden, J. A. Aflatoxin inhibition and fungistasis by peanut tannins. Peanut Science. 9, 17-20 (1982).

65. Yen, G. C., Duh, P. D., \& Tsai, C. L. Relationships between antioxidant activity and maturity of peanut hulls. Journal of Agricultural and Food Chemistry. 41, 67-70 (1993). 